\title{
Parameter Optimization of ARQ System based on Proximity-1 Protocol
}

\author{
Jie Wang ${ }^{1, a}$ Chenghua Wang ${ }^{1, b}$ Hao $\mathrm{Wu}^{1, \mathrm{c}}$ and Qiangqiang Mao ${ }^{1, \mathrm{~d}}$ \\ ${ }^{1}$ College of Electronic and Information Engineering, Nanjing University of Aeronautics and \\ Astronautics, Nanjing 211106, China
}

\author{
awj1391992076@163.com, bchwang@nuaa.edu.cn, wuhao0408102@foxmail.com, \\ d844628006@qq.com
}

\section{Keywords: CCSDS; Proximity-1 protocol; GBN-ARQ; Frame length; Sliding window length}

\begin{abstract}
In order to improve the communication efficiency of the spacecraft in the Proximity space link, a GBN-ARQ scheme is designed based on the proximity-1 protocol. On the basis of this, to maximize the system throughput and normalized throughput, the data frame length and the sliding window length are optimized, and their theoretical optimal value are obtained. Simulation and analysis results show that the data frame length of the ARQ scheme should be set to 1024byte, and the system efficiency can be improved by selecting the appropriate window length in the given system parameters.
\end{abstract}

\section{Introduction}

With the development of deep space exploration technology, the single spacecraft mission model has become the history of the past. In order to adapt to multi-spacecraft missions, the Consultative Committee for Space Data System (CCSDS) has proposed a new system, the proximity-1 Space Link Protocol[1,2], on the basis of the existing Conventional Orbit Systems (COS) and Advanced Orbit Systems (AOS). It is suitable for short-range, two-way, fixed or mobile deep-space communication links and is usually used in the mutual communication between detectors, orbit satellites, relay satellites and planetary landers $[3,5]$.

In the deep space exploration task, because of the occlusion, absorption, reflection, folding and diffraction caused by various objects on the surface of planet, the signal from the transmitter will form a plurality of path signal components to reach the receiving end. The signal components of different paths have different phase, amplitude and propagation delay, moreover, the channel noise is added, and the superposition of the received signals can cancel and enhance each other. In addition, most of the detector is in the state of motion relative to the track, it will cause the channel characteristics change with time, and the received signal will produce more serious distortion due to the Doppler Effect [6]. In order to ensure effective and reliable data transmission, the design of Proximity-1 protocol frame structure is compatible with the transmission mechanism of automatic retransmission. However, if the ARQ transmission mechanism is used, the system throughput will decrease rapidly and the system delay will increase when the proximity- 1 space link becomes worse and the bit error rate increases. Therefore, how to design the ARQ system scheme and the optimization of transmission parameters, to ensure the automatic retransmission function, throughput and delay performance, has become the key issue in the design of the space communication system.

For the ground mobile communication system, the theoretical analysis model of ARQ system throughput and delay has been established. As shown in the literature [7], the throughput performance of the adaptive ARQ is studied, and it is proved that the multi copy ARQ has better throughput performance at high channel error rate. Literature [8] proposes a new GBN-ARQ algorithm, which is suitable for TDD time-varying slow fading system, and can improve the efficiency of the system when the throughput is low. Based on the assumption that the feedback signal can be correctly received, the probability generating function of GBN-ARQ system queue length and waiting time distribution is derived in literature [9]. None of the above studies are related to the effect of the frame length and the length of the sliding window on the performance of the 
system. In this paper, the optimal frame length and the minimum sliding window length are obtained based on the maximum throughput of the system and the requirement of the system error rate.

\section{ARQ Scheme based on Proximity-1 Protocol}

Protocol Transport Unit. The proximity-1 protocol transfers data and control instructions in the Proximity Link Transmission Unit [10] (PLTU) (refer with: Fig. 1). It should be emphasized that the frame structure retains the definition of the acknowledgement signal (ACK) and the negative acknowledgement signal (NACK). In particular, the PLTU includes a 24bit additional synchronization identifier (FAF320H), a 32bitCRC checksum, and a transmission frame of up to 2048bit. An additional synchronization identifier is used to identify the transmission frame at the receiving end. The CRC checksum is used to detect the error of the transmission frame. The data domain in the transmission frame can carry user-defined data and transmit feedback signals. The value of the retransmission identifier determines whether the feedback signal is ACK or NACK, and the value of the report indicates the frame number of the feedback.

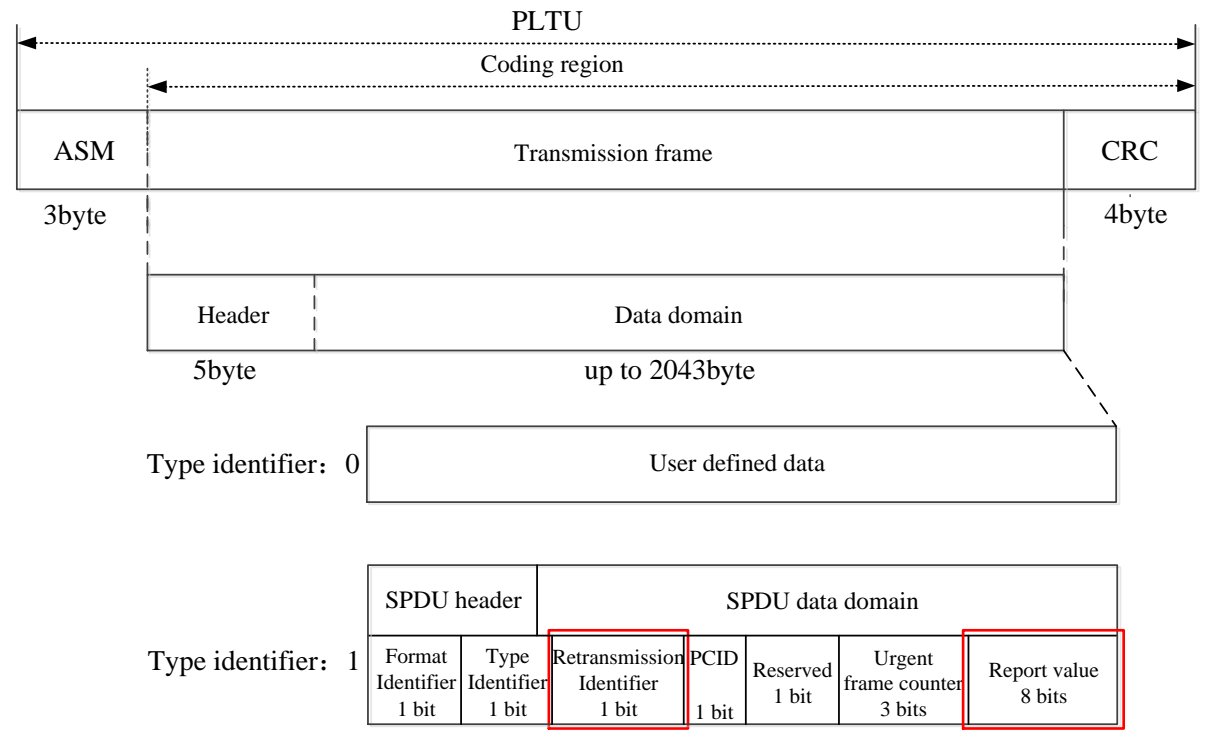

Figure 1. Proximity link transmission unit

Design of ARQ Scheme. The traditional ARQ scheme including the stop-and-wait (SW), th go-back-N (GBN), and selective repeat (SR). Among them, the SW-ARQ is poor in the channel utilization performance, and the system throughput is relatively small. The transmission efficiency of SR-ARQ is very high, but at the expense of the complex receiver design. The transmission efficiency of GBN-ARQ is amount to SR-ARQ, and the realization of the receiver is relatively simple. Combined with the characteristics of deep space communication environment and Proximity-1 protocol, we choose the GBN scheme. The specific implementation process is shown in Fig. 2. 


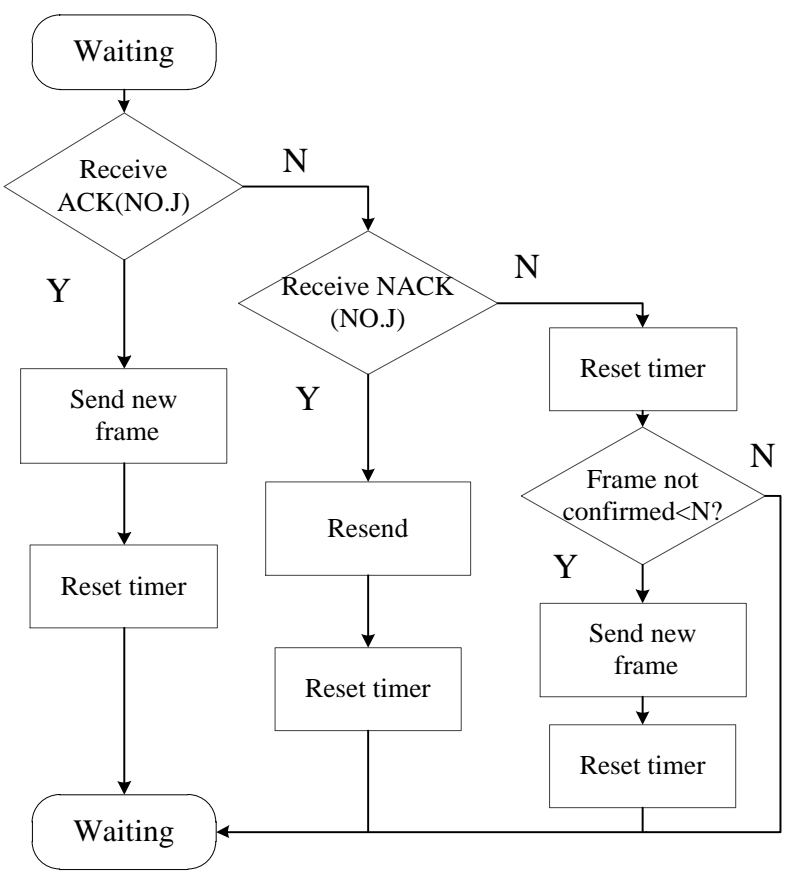

(a) The sender process

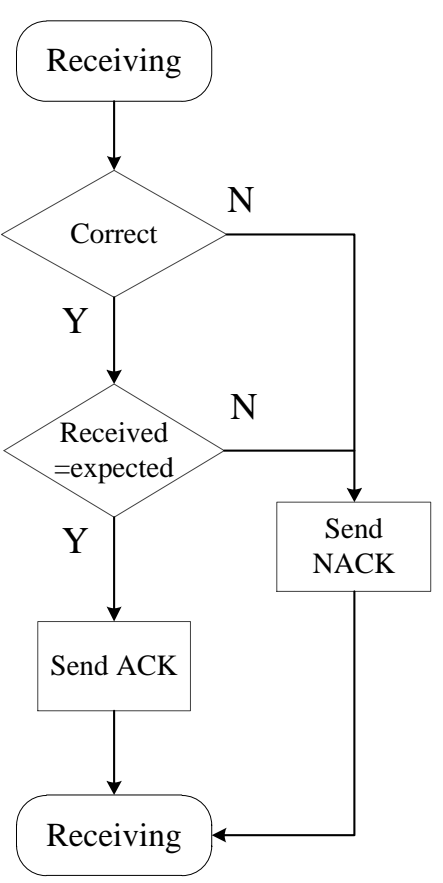

(b) The receiver process

Figure 2. GBN-ARQ scheme based on Proximity-1 protocol

Fig. 2 (a) shows that the sender needs to deal with three types of events in the process of waiting for the feedback signal. When receiving ACK feedback, the sender determines whether the feedback frame number is within the range of the sliding window. If it exists, moving sliding window to the next frame position, sending a new data frame, and reset the timer. When a NACK feedback is received, the frame and its subsequent $\mathrm{N}-1$ data frames are retransmitted. If the timer is out of time and the number of frames is less than $\mathrm{N}$, the sender sends a new transmission frame, otherwise continue to wait for the feedback signal. For the receiver, only when receiving a correct frame and the frame number is equal to the expected number, does it send a ACK signal.

\section{Parameter Optimization of ARQ System}

Optimization of Frame Length. Throughput is one of the important parameters to evaluate the performance of communication systems. When the frame format and the channel error rate are determined, if the frame length is small, the frame head occupies a large proportion of the data transmission unit, the payload data is small, resulting in low throughput. On the other hand, if the frame length increases, the probability of dropping frames will increase, which will lead to the decrease of throughput too. According to the definition, the throughput of the Proximity-1 ARQ system [11] is

$$
\eta=\frac{n-l^{\prime}}{n+l^{\prime \prime}} \cdot p_{c}
$$

Wherein, $l^{\prime}$ is frame header length, $l^{\prime \prime}$ is the length of ASM and CRC, as shown in Fig. 1, $l^{\prime}=5, l^{\prime \prime}=7$. Total transmission frame length $n=l+l^{\prime}, l$ is data field length, $p_{c}$ is the probability of sending the correct frame.

For Proximity-1 data services, every bit is independent of each other. So the relationship between frame error rate and bit error rate is

$$
p_{f}=1-\left(1-p_{e}\right)^{\left(n+l^{\prime \prime}\right) \times 8}
$$




$$
p_{c}=1-p_{f}=\left(1-p_{e}\right)^{\left(n+l^{\prime \prime}\right)[8}
$$

Fig. 3 shows a typical case $\left(p_{e}=10^{-5}, 10^{-6}, 10^{-7}\right)$ where the throughput of the system varies with the frame length $\mathrm{n}$. Under the condition of different channel bit error rate, the changes of the throughput are relatively slow near the optimal frame length, and the frame length is similar. When the frame length is 1024 byte, it can be seen that under the condition of high bit error rate $\left(10^{-5}\right)$, the difference between throughput and optimal throughput is $2.9 \%$. And the difference changes to $0.6 \%$, under the condition of low bit error rate $\left(10^{-7}\right)$. According to the above analysis, the frame length in actual Proximity-1 ARQ system can be set to 1024byte.

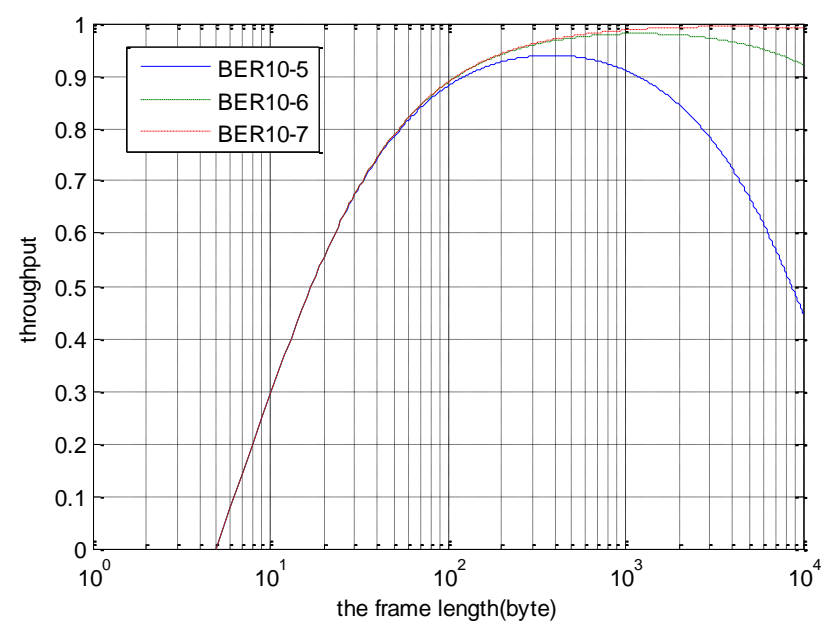

Figure 3. System throughput versus frame length

Optimization of Sliding Window Length. The actual delay of sending a data frame includes a one-way transmission delay $T_{1}$, the resending delay $T_{r}$ (including the propagation and procession delay $t_{b}$, and the receiver feedback delay $t_{f}$ ). All the frames arrive at the queue independently according to the Poisson process with rate $\lambda$. Therefore the service delay can be considered an equivalent service delay, which follows a general distribution and can be described as an M/1/G/ queuing model[12]. The expression of mean waiting delay of GBN-ARQ is

$$
W=\frac{R}{1-\rho}=\frac{\lambda \overline{X^{2}}}{2(1-\rho)}(\rho=\lambda \bar{X})
$$

Wherein, $X_{k}$ is the $k$ th frame equivalent service delay, $\bar{X}$ is the first moments of equivalent service delays.

We assume a resented packet is equiprobably located in every sliding window ( $n$ packets). So the mean frame service delay in the first transmission at the receiver can be calculated as

$$
T_{1}=m[n+(n-1)+\ldots+2+1] / n=[m(n+1)] / 2
$$

We suppose each frame is successfully transmitted with probability $p$. When a frame is retransmitted for $k$ times, the equivalent retransmission delay $T_{r}$ is

$$
T_{r}=m k n+(k+1)\left(t_{b}+t_{f}\right)
$$

That is, the probability distribution of stochastic variable $X_{k}$ is 
$P\left[X_{k}=\frac{m(n+1)}{2}+m k n+(k+1)\left(t_{b}+t_{f}\right)\right]=p(1-p)^{k}$

The first and the second moments of service delays are

$$
\begin{aligned}
\bar{X} & =\sum_{k=0}^{\infty}\left[\frac{m(n+1)}{2}+m k n+(k+1)\left(t_{b}+t_{f}\right)\right] p(1-p)^{k} \\
= & \frac{m(n+1)}{2}+\frac{m n(1-p)}{p}+\frac{t_{b}+t_{f}}{p} \\
\overline{X^{2}} & =\sum_{k=0}^{\infty}\left[\frac{m(n+1)}{2}+m k n+(k+1)\left(t_{b}+t_{c}\right)\right]^{2} p(1-p)^{k} \\
& =\left[\frac{m(n+1)}{2}+t_{b}+t_{f}\right]^{2}+\frac{\left[2\left(t_{b}+t_{f}\right)+m(n+1)\right]\left[t_{b}+t_{f}+m n\right](1-p)}{p} \\
& +\frac{\left[m n+t_{b}+t_{f}\right]^{2}(1-p)(2-p)}{p^{2}}
\end{aligned}
$$

Inserting Eq. 6 and Eq. 7 into Eq. 4, we obtain the mean frame service delay for GBN-ARQ system.

$$
\begin{array}{r}
T=\bar{X}+W=\frac{m(n+1)}{2}+\frac{m n(1-p)+\left(t_{b}+t_{f}\right)}{p} \\
+\frac{\lambda\left[\mathrm{K}_{1}+\frac{K_{2}(1-p)}{p}+\frac{K_{3}(1-p)(2-p)}{p^{2}}\right]}{2\left\{1-\lambda\left[\frac{m(n+1)}{2}+\frac{m n(1-p)+\left(t_{b}+t_{f}\right)}{p}\right]\right\}}
\end{array}
$$

Wherein

$$
K_{1}=\left[\frac{m(n+1)}{2}+t_{b}+t_{f}\right]^{2}, \quad K_{2}=\left[2\left(t_{b}+t_{f}\right)+m(n+1)\right]\left[t_{b}+t_{f}+m n\right], \quad K_{3}=\left[m n+t_{b}+t_{f}\right]^{2}
$$

When the system is saturated, the normalized maximum throughput is

$$
\rho_{\max }=\frac{1}{T} \sqcup\left(T_{1}+t_{b}+t_{f}\right)=\frac{m(n+1)+2\left(t_{b}+t_{f}\right)}{2 T}
$$

According to Eq. 9, we can obtain the optimal sliding window length in the given system.

$$
n=f\left(m, \lambda, p, t_{b}, t_{f}\right)
$$

Fig. 4 and Fig. 5 give the simulation analysis results about the mean frame service delay, the normalized maximum throughput, and the optimal sliding window length(Here assuming $\lambda=0.001, m=4(1024$ byte $\left.), t_{b}=t_{f}=1.5 \mathrm{~ms}\right)$. 


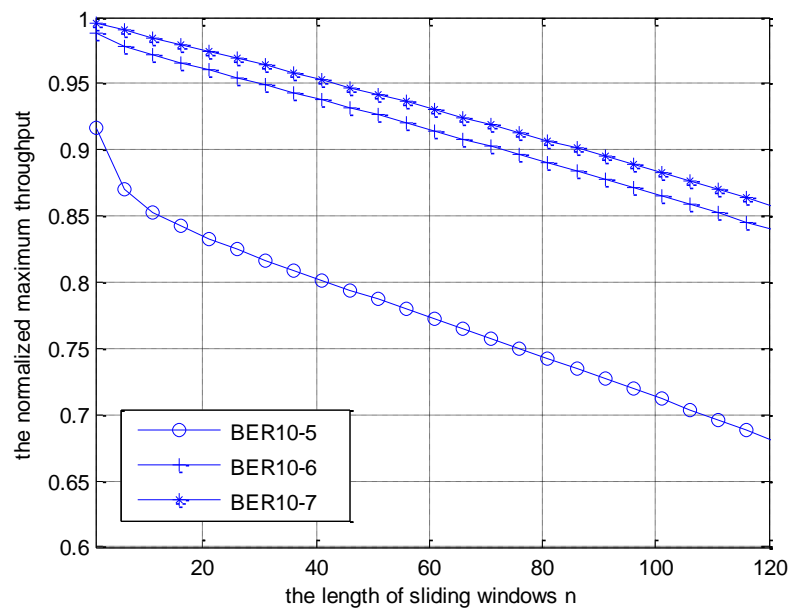

Figure 4. Sliding window length versus normalized throughput

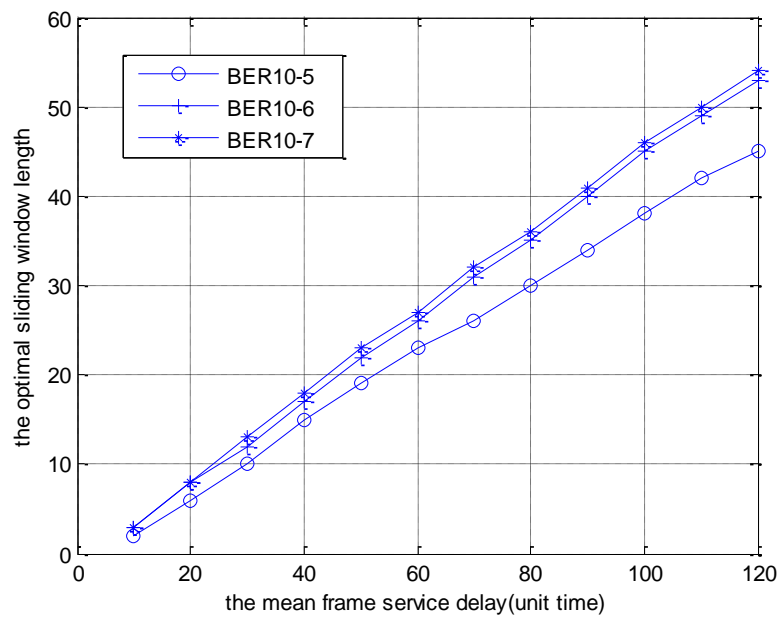

Figure 5. Mean frame service delay versus optimal sliding window length

When the system parameters are given, the normalized throughput decreases with the increase of the sliding window length, especially in the case of high bit error rate. In order to achieve the same normalized throughput, we can reduce the sliding window length. When the mean frame service delay is accurate, the optimal sliding window length of the system can be determined (the solution of Eq. 10 exists), and the sliding window length decreases with the increase of bit error rate.

\section{Summary}

This paper is based on CCSDS Proximity-1 protocol, we design automatic retransmission scheme based on GBN-ARQ. The length of the data frame and the sliding window length are optimized from the view of improving the throughput performance of the system. The results show that the system efficiency can be improved by choosing the appropriate frame length and sliding window length when given the system parameters. This provides a good theoretical guidance to improve the system algorithm and system efficiency.

\section{References}

[1] F. Ren, H.P. Zhao and X. Chen. Strategy Study of Dynamic Variable Frame Length Based on CCSDS Proximity-1 Protocol. Spacecraft Engineering, Vol. 22(2013) No.4, p.72 (In Chinese).

[2] N. Wang, J.H. Zhang and G.H. Zhang. The Overviews on Proximity-space Link Protocol. Space Electronic Technology, (2011)51-55 (In Chinese).

[3] CCSDS. 211.0-B-5 Promixity-1 space link protocol-data link layer. Recommendation for space data system standards. Washington D.C: CCSDS, (2013)

[4] CCSDS. 211.2-B-2 Promixity-1 pace link protocol-coding and synchronization layer. Recommendation for space data system standards. Washington D.C: CCSDS, (2013)

[5] CCSDS. 211.1-B-4 Promixity-1 space link protocol-physical layer. Recommendation for space data system standards. Washington D.C: CCSDS, (2013)

[6] J.R. Agrc, I.P. Clare. Advanced communication and networking technologies for Mars exploration. (2012).

[7] A. Mehta, D. Kagaris and R. Viswanathan. Throughput Performance of an Adaptive ARQ Scheme in Rayleigh Fading Channels. IEEE Transactions on Wireless Communications. Vol. 5(2006) No.1, p.12. 
[8] B. He, J. Li and J.R.Lin. Adaptive Predicted Repeat GBN-ARQ Algorithm in TDD Slowly Fading Channel. Journal of Electronics \& Information Techology, (2006 ), No.11, p. 2086 (In Chinese).

[9] M. Yoshimoto, T. Takine, Y. Takahashi, et al. Waiting time and queue length distributions foe go-back-N and selective-repeat ARQ protocols. IEEE Transactions on Communications, Vol. 41(1993) No.11, p. 1687.

[10]H.J. Zhang, J. Guo. Research on Application of Proximity-1 Space Link Protocol in Mars Exploration. Spacecraft Engineering, Vol. 24(2015) No.2, p.75 (In Chinese).

[11] G. Benelli. A Go-Back-N protocol for mobile. IEEE Transactions on Vehicular Technology, Vol. 40(1991) No.4, p.714.

[12] S.P. Li, C.M. Liu and Z.P.He. Research on the Delay Performance of GBN-ARQ and SR-ARQ Systems in Wireless Data Transmission. Signal Processing, (2009)384-388 (In Chinese). 